\title{
Prevalence of Pre-End-Stage Renal Disease Care and Associated Outcomes among Urban, Micropolitan, and Rural Dialysis Patients
}

\author{
Saugar Maripuri T. Alp Ikizler Kerri L. Cavanaugh \\ Division of Nephrology and Hypertension, Department of Medicine, Vanderbilt University Medical Center, \\ Nashville, Tenn., USA
}

\section{Key Words}

Rural patients · Disparity · Chronic kidney disease

\begin{abstract}
Background/Aims: Pre-end-stage renal disease (ESRD) care is associated with improved outcomes among patients receiving dialysis. It is unknown what proportion of US micropolitan and rural dialysis patients receive pre-ESRD care and benefit from such care when compared to urban. Methods: A retrospective cohort study was performed using data from the US Renal Data System. Patients $\geq 18$ years old who initiated dialysis in 2006 and 2007 were classified as rural, micropolitan or urban and the prevalence of pre-ESRD care (early nephrology care $>6$ months, permanent vascular access, dietary education) was determined using the medical evidence report. The association of pre-ESRD care with dialysis mortality and transplantation was assessed using Cox regression with stratification for geographic residence. $\boldsymbol{R e}$ sults: Of 204,463 dialysis patients, $80 \%$ were urban, $10.2 \%$ were micropolitan and $9.8 \%$ were rural. Overall attainment of pre-ESRD care was poor. After adjustment, there were no significant geographic differences in attainment of early nephrology care or permanent dialysis access. Receiving care reduced all-cause mortality and increased the likelihood of transplantation to a similar degree regardless of geographic residence. Both micropolitan and rural patients received less dietary education (relative risk $=0.80,95 \% \mathrm{Cl}=0.76-0.84$ and relative risk $=0.85,95 \% \mathrm{Cl}=0.80-0.89$, respectively). Conclu-
\end{abstract}

\section{KARGER}

E-Mail karger@karger.com

www.karger.com/ajn sion: Among patients who receive dialysis, the prevalence of early nephrology care and permanent dialysis access is poor and does not vary by geographic residence. Micropolitan and rural patients receive less dietary education despite an observed mortality benefit, suggesting that barriers may exist to quality dietary care in more remote locations.

Copyright $\odot 2013$ S. Karger AG, Basel

Health outcomes among patients living in remote locations is of increasing focus in the United States, as approximately $20 \%$ of the population live in micropolitan (small towns) or rural areas [1]. Among end-stage renal disease (ESRD) patients receiving dialysis, micropolitan and rural residence are independently associated with worse mortality in more remote patients, particularly those on peritoneal dialysis [2]. It is unknown if lack of quality of pre-ESRD care could partially explain this increased risk.

Optimal pre-ESRD care includes timely referral to a nephrologist, dialysis and dietary education, placement of a permanent vascular access in patients who prefer hemodialysis, and referral for pre-emptive kidney transplantation $[3,4]$. Pre-ESRD nephrology care has been independently associated with decreased dialysis mortality, higher likelihood of pre-emptive kidney transplantation, higher serum albumin concentrations at initiation of dialysis, and higher incidence of arteriovenous fistula or graft use for hemodialysis initiation [5-9]. Timely referral

Kerri L. Cavanaugh, MD, MHS

Division of Nephrology and Hypertension, Vanderbilt University Medical Center 116121 st Avenue MCN S-3223

Nashville, TN 37232 (USA)

E-Mail kerri.cavanaugh@vanderbilt.edu 


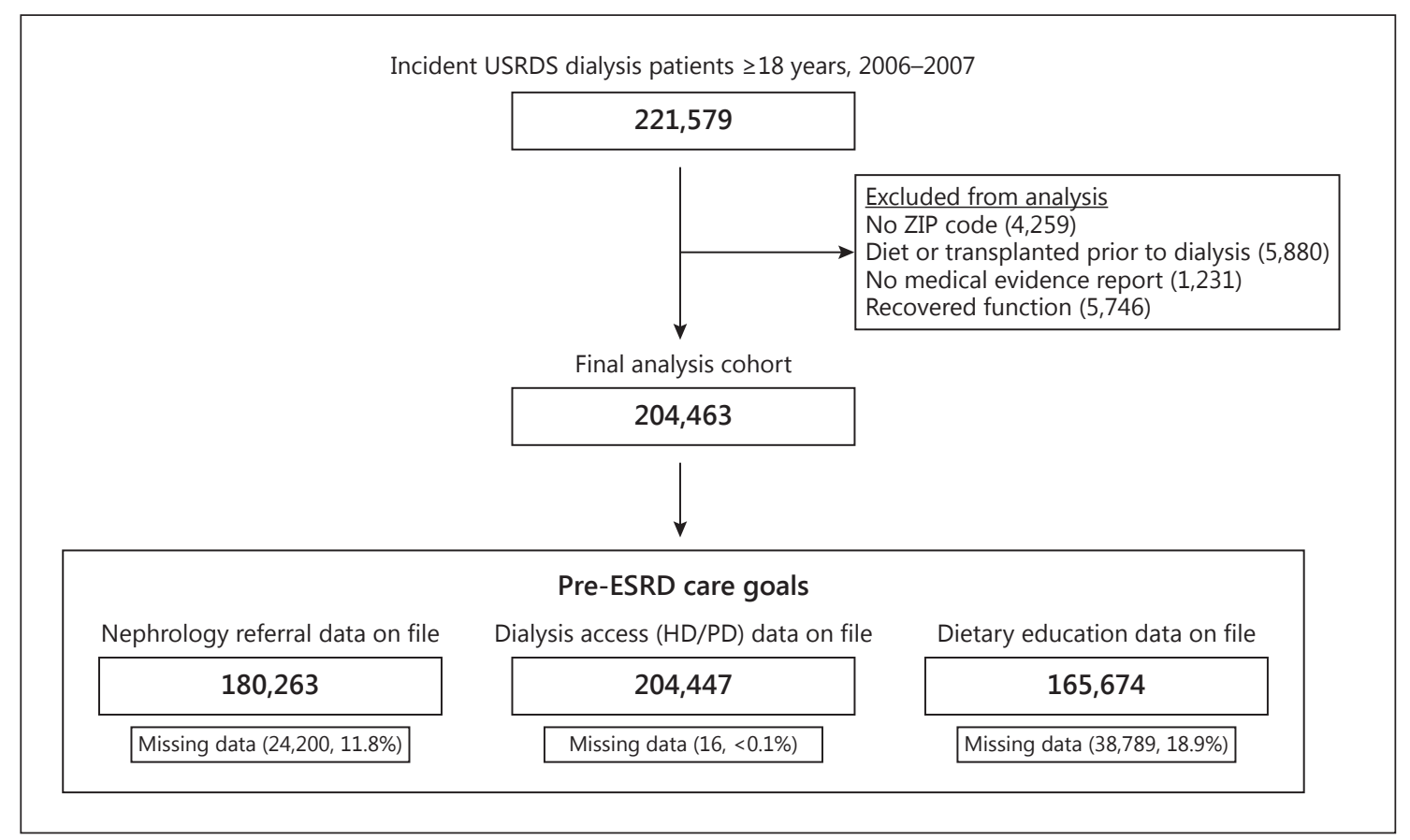

Fig. 1. Study flow.

and quality care prior to the start of dialysis has been identified as an area requiring improvement in the United States [10].

Micropolitan and rural communities face barriers in receiving specialized care, which may be related to lack of local subspecialists and hospitals with advanced resources [11]. These barriers may limit access to nephrology support required for optimum pre-ESRD care. This study examines the prevalence of pre-ESRD care among a population of urban, micropolitan and rural dialysis patients and its impact on mortality and kidney transplantation. We hypothesized that micropolitan and rural residence would associate with lower prevalence of pre-ESRD care and reduce the protective effect of such care.

\section{Materials and Methods}

A retrospective cohort study was performed using patient-level data obtained from the US Renal Data System (USRDS). The design of the study cohort including data sources, patient selection, and determination of rural location has previously been described in more detail [2]. This study was approved by the Institutional Review Board at Vanderbilt University Medical Center and by the US Renal Data System.

Rural Disparities in Pre-ESRD Care

\section{Patient Selection}

Patients were included if they initiated dialysis for the first time between January 1, 2006 and December 31, 2007 and were 18 years of age or older. Patients were excluded if their residence location could not be determined, the medical evidence report was missing, or they discontinued dialysis during follow-up due to recovery of kidney function. Figure 1 depicts the study flow.

\section{Identification of Residence Location}

Rural and micropolitan residence was determined by the use of rural-urban commuting area codes [12], a robust and flexible method for measuring the degree of rurality in epidemiologic research [13]. ZIP code-specific rural-urban commuting area codes were used as they represent the smallest feasible geographic area to study [14].

\section{Pre-ESRD Care Goals}

The prevalence of selected pre-ESRD care goals was determined from the medical evidence report, including: (1) early nephrology care $>6$ months prior to dialysis initiation (yes/no), (2) mature permanent hemodialysis access (arteriovenous fistula or graft) at initiation (yes/no), and (3) dietary education prior to dialysis initiation (yes/no).

\section{Mortality and Kidney Transplantation}

Longitudinal outcomes of interest included time to death and time to kidney transplantation as reported from the first dialysis service date. For survival analysis, patients who received a transplant were censored at the time of their kidney transplant. For transplantation analysis, patients were excluded from the 'at risk' 
population if they were $>75$ years old at initiation or were deemed unfit for transplant on the medical evidence report. Censoring occurred at the date of the outcome, last follow-up or October 1, 2009, whichever occurred earlier.

\section{Adjustment Covariates}

Demographic information included age at initiation, sex, and race. Measures of socioeconomic status that are available in the USRDS, such as insurance coverage and employment status, were included. Ecologic surrogates from the US Census were utilized for measures not collected by the USRDS, including ZIP code median household income and ZIP code proportion over age 25 with a high school diploma [15]. Characteristics of kidney disease included reported cause of chronic kidney disease (CKD), estimated glomerular filtration rate (eGFR) at initiation, and dialysis modality at 90 days after initiation. Covariates describing chronic medical comorbidities were included such as heart failure, coronary artery disease, diabetes mellitus, hypertension, chronic obstructive pulmonary disease, cancer, history of stroke, and use of tobacco products, alcohol or illicit drugs. Documented history of institutionalization and impairment of activities of daily living were also included as they could impede patients from seeking medical care.

\section{Statistical Analysis}

Baseline characteristics were summarized as percentages, medians with interquartile ranges, or means with standard deviations, as appropriate based on the variable type and frequency distribution. Comparisons across geographic (urban, micropolitan, and rural) groups were made using Pearson's $\chi^{2}$ test for categorical and the Kruskal-Wallis test for continuous data due to nonnormal distributions. Statistical significance was defined at a $p$ value $<0.05$ and all tests were two-tailed.

The unadjusted prevalence of pre-ESRD care by geographic residence was calculated. Adjusted estimates were determined through Poisson regression with robust variances, which provides relative risk (RR) estimates and correct confidence intervals [16]. The unadjusted association of pre-ESRD care with longitudinal outcomes was assessed using the Kaplan-Meier method for time to death and time to transplantation with stratification by geographic residence. Multivariable Cox regression models were created with stratification for geographic location (urban, micropolitan, rural). Stratified analyses were performed due to the a priori hypothesis that differential effects may be present in different geographic strata.

Poisson and Cox regression models were adjusted for demographics (age, sex, race), body mass index, US region (by ESRD network: northeast $1-5$, midwest $9-12$, south 6-8 and 13-14, and west 15-18), socioeconomic status (insurance, employment, ZIP code median household income), kidney disease-related factors (presumed primary cause of ESRD, eGFR at initiation) and comorbidities (as listed in 'covariates of interest'). All Cox models were tested for the proportional hazard assumption through use of loglog survival plots and were found to satisfy the assumption.

As depicted in figure 1, substantial missing data were observed in the documentation of nephrology care (11.3\% missing) and dietary education $(20.1 \%$ missing). The characteristics of missing data were assessed for nonrandomness using pairwise correlation matrices. There was no independent correlation between missing data in pre-ESRD care variables (nephrology care, permanent vascular access and dietary education) and other variables in the analysis (correlation coefficients $<0.1$ ), suggesting that missing data were mostly random. Missing data were handled through the use of listwise deletion for the multivariable models, with the analysis only including patients with complete data on file. Other covariates were rarely missing $(<1 \%)$. Documentation of survival and transplantation was based entirely on data from the USRDS.

Data management and statistical analysis were performed using Stata version 11.2 (College Station, Tex., USA).

\section{Results}

\section{Cohort Characteristics}

Baseline characteristics of this cohort are summarized in table 1. Micropolitan and rural patients were modestly older than urban patients, lived in less wealthy communities, and had fewer high school graduates compared to urban communities. Blacks accounted for only $21.6 \%$ of micropolitan and $21.1 \%$ of rural patients compared to $31.4 \%$ of urban patients. Micropolitan and rural patients had a higher prevalence of heart failure, heart disease, stroke, hypertension, diabetes mellitus, and chronic obstructive pulmonary disease.

\section{Prevalence of Pre-ESRD Care}

The overall prevalence of pre-ESRD care was poor in the study cohort (nephrology care $53.5 \%$, permanent dialysis access $17.7 \%$ and dietary education $11.9 \%$ ). Table 2 summarizes the prevalence of pre-ESRD care by geographic residential location. The unadjusted prevalence and adjusted RR of early nephrology care and permanent dialysis access (among hemodialysis patients) was similar across geographic groups. Dietary education prior to initiation of dialysis was significantly less likely to occur among micropolitan $(\mathrm{RR}=0.80,95 \% \mathrm{CI}=0.69-0.93)$ and rural patients $(\mathrm{RR}=0.85,95 \% \mathrm{CI}=0.73-0.98)$. Patients who received dietary education had significantly higher serum albumin at the time of initiation $(3.30 \mathrm{~g} / \mathrm{dl}$ with $\mathrm{SD}=0.70)$ compared to those without dietary education $(3.11 \mathrm{~g} / \mathrm{dl}$ with $\mathrm{SD}=0.72 ; \mathrm{p}<0.001)$. The serum albumin among patients who received dietary education did not vary by geographic region (urban: $3.30 \mathrm{~g} / \mathrm{dl}$ with $\mathrm{SD}=$ 0.69; micropolitan: $3.29 \mathrm{~g} / \mathrm{dl}$ with $\mathrm{SD}=0.70$; rural: $3.26 \mathrm{~g} /$ dl with $\mathrm{SD}=0.71 ; \mathrm{p}=0.12$ ).

\section{Dialysis Mortality and Likelihood of Kidney Transplantation}

The association of pre-ESRD care with mortality and kidney transplantation was examined. Table 3 summarizes the multivariable Cox models for the association of pre-ESRD care with dialysis mortality and kidney transplantation, stratified by geographic residence. Early ne- 
Table 1. Baseline characteristics of the study group

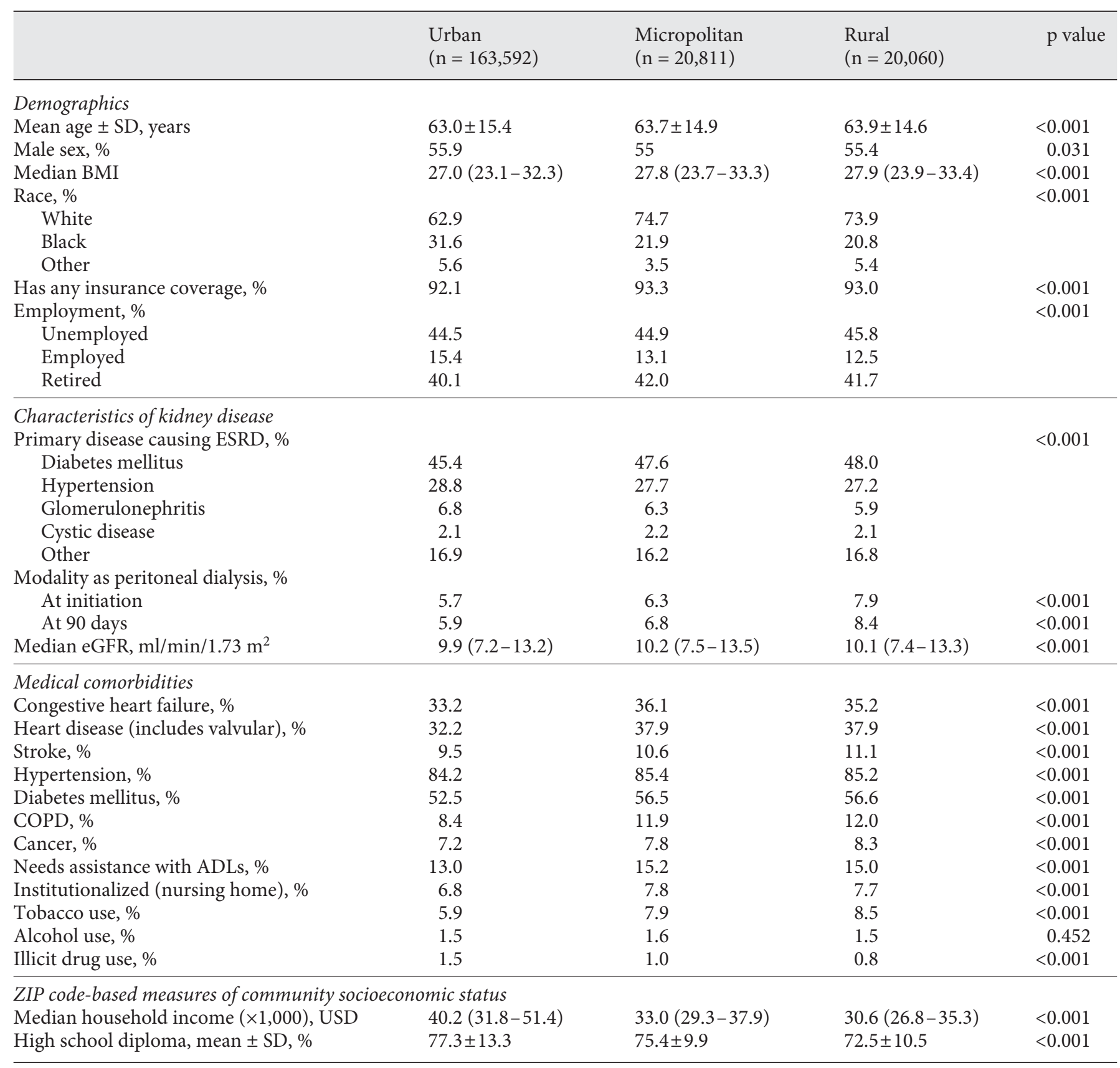

Statistical testing performed by Pearson's $\chi^{2}$ test for categorical variables and the Kruskal-Wallis test for continuous variables. Standard deviation and 25-75th interquartile ranges (in parentheses) are reported for means and medians, respectively. Estimates of ZIP code income and education are based on calculations from the US Census, 2000. COPD = Chronic obstructive pulmonary disease; ADLs = activities of daily living.

phrology care (hazard ratio for death $=0.79,95 \% \mathrm{CI}=$ $0.78-0.80$ ), permanent dialysis access (hazard ratio for death $=0.63,95 \% \mathrm{CI}=0.62-0.65)$, and dietary education (hazard ratio for death $=0.90,95 \% \mathrm{CI}=0.88-0.92$ ) are all associated with decreased mortality with no evidence of effect modification by geographic strata. Early nephrology care (hazard ratio for transplant $=1.45,95 \% \mathrm{CI}=$ 1.39-1.52) and dietary education (hazard ratio for trans- 
Table 2. Prevalence of pre-ESRD care goals by geographic location

\begin{tabular}{|c|c|c|c|}
\hline & Urban & Micropolitan & Rural \\
\hline \multicolumn{4}{|c|}{ Nephrologist care $>6$ months prior to initiation of dialysis } \\
\hline Prevalence, \% & 46.8 & 45.3 & 46.1 \\
\hline Unadjusted & 1.00 (reference) & $1.03(1.01-1.04)$ & $1.01(0.99-1.03)$ \\
\hline Adjusted & 1.00 (reference) & $1.02(0.98-1.06)$ & $1.02(0.97-1.07)$ \\
\hline \multicolumn{4}{|c|}{ Mature permanent dialysis access ( $A V F$ or $A V G$ ) used upon initiation of hemodialysis } \\
\hline Prevalence, \% & 17.7 & 17.8 & 17.9 \\
\hline Unadjusted & 1.00 (reference) & $1.01(0.98-1.04)$ & $1.01(0.98-1.05)$ \\
\hline Adjusted & 1.00 (reference) & $1.03(0.95-1.10)$ & $1.04(0.96-1.12)$ \\
\hline \multicolumn{4}{|c|}{ Dietary education received prior to initiation of dialysis } \\
\hline Prevalence, \% & 12.4 & 9.5 & 10.1 \\
\hline Unadjusted & 1.00 (reference) & $0.76(0.73-0.80)$ & $0.81(0.77-0.85)$ \\
\hline Adjusted & 1.00 (reference) & $0.80(0.69-0.93)$ & $0.85(0.73-0.98)$ \\
\hline
\end{tabular}

Relative risks by Poisson regression (95\% CIs). Multivariable models adjusted for age, sex, race, BMI, insurance coverage, employment status, ZIP code median household income, presumed primary cause of CKD, eGFR at initiation, modality choice at 90 days after initiation of dialysis, and medical comorbidities.

Table 3. Association of pre-ESRD goal and long-term outcomes with stratification by geographic location

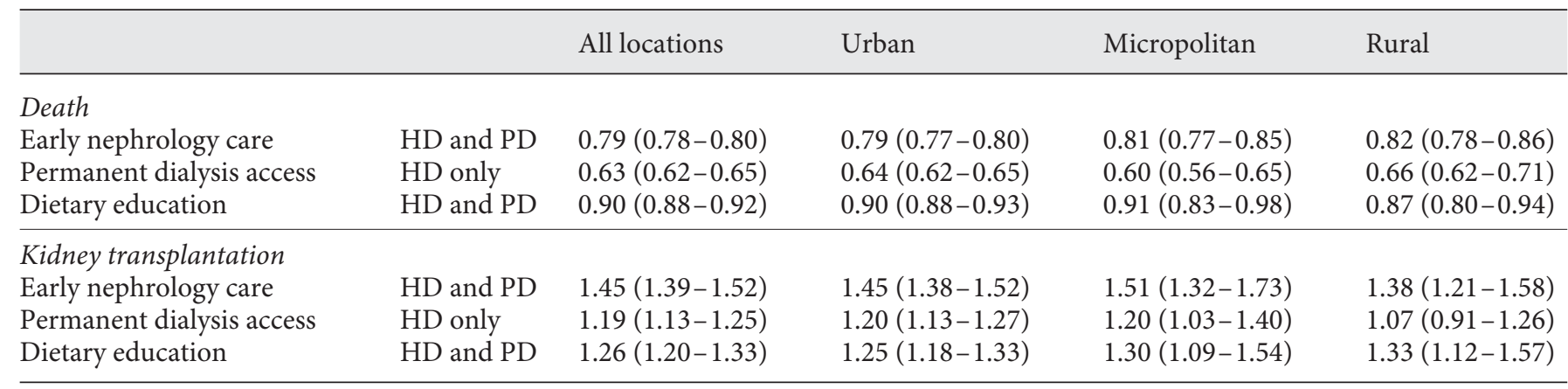

Hazard ratios by Cox regression (95\% CIs). Multivariable models adjusted for age, sex, race, BMI, insurance coverage, employment status, ZIP code median household income, dialysis modality at 90 days, presumed primary cause of CKD, eGFR at initiation, and medical comorbidities. $\mathrm{HD}=$ Hemodialysis; $\mathrm{PD}=$ peritoneal dialysis.

plant $=1.26,95 \% \mathrm{CI}=1.20-1.33)$ both increase the likelihood of kidney transplantation, an effect that is also demonstrated at similar magnitude across geographic strata. All tests for interaction (comparison between strata) were not statistically significant $(\mathrm{p}>0.10)$.

\section{Discussion}

Micropolitan and rural dialysis patients obtained early nephrology care and permanent hemodialysis access at comparable rates as urban patients and shared a similar reduction in mortality from such care, suggesting that geographic residence does not substantially impact basic pre-ESRD care in the US dialysis population. The notable exception is dietary education, where micropolitan and rural dialysis patients receive less pre-ESRD dietary care.

Previous studies focusing on rural health have described barriers that may preclude quality care in the micropolitan or rural setting. This includes dependence on community health centers, challenges in subspecialty access [17], clustering of providers in urban areas [18], and lack of access to transportation [19]. A retrospective study in Canada by Tonelli and colleagues [18] found that re- 
mote-dwelling CKD patients, defined as having an eGFR $<45 \mathrm{ml} / \mathrm{min}$, were less likely to be referred to a nephrologist and have quality CKD care. In addition, remote patients were more likely to die during follow-up, presumably from higher cardiovascular risk and limitations in health care access in rural Canadian locales. In contrast, our study of US dialysis patients suggests that micropolitan and rural patients obtain pre-ESRD care from nephrologists and dialysis access surgeons at a similar prevalence as those in urban communities. A number of factors may influence these disparate findings. First, our study focused on dialysis patients who survived advanced $\mathrm{CKD}$ to initiate dialysis and not the general CKD population. Second, advanced CKD, often with an eGFR $<20 \mathrm{ml} /$ min, is associated with significant morbidity and mortality requiring subspecialty and multidisciplinary care. In such circumstances, the proximity to needing dialysis treatments may trump physical remoteness as a barrier to access of pre-ESRD care. Third, dialysis unit penetration into rural areas has advanced over time, allowing increasing access to nephrology services while maintaining similar facility outcomes when compared to urban units [20]. Lastly, it is important to recognize that the overall prevalence of basic pre-ESRD care in the United States is exceedingly low regardless of geographic location, which makes detecting differences between regions less likely and highlights the need for systematic improvements throughout the nation.

Despite the lack of differences in nephrology care and permanent access placement, micropolitan and rural dialysis patients had less pre-ESRD dietary care compared to urban. Pre-ESRD dietary education is recommended for all patients with advanced CKD [21]. It is a complex task that requires attention to nutrition, fluid and protein intake, sodium, potassium, and phosphorus. Counseling can improve the management of hyperkalemia, hyperphosphatemia, and avoidance of protein-energy wasting $[21,22]$. Dietary education is associated with lower dialysis mortality, a finding that was first noted in another cohort of USRDS patients by Slinin et al. [23] and corroborated in this study population. Patients who receive preESRD dietary education initiate dialysis with higher serum albumin that does not vary with geographic residence. This suggests that receipt of dietary education confers similar benefit regardless of where patients live.

The prevalence of pre-ESRD dietary education was low, suggesting that barriers to dietary education exist in all geographic strata but are more pronounced in micropolitan and rural areas. The mechanisms behind this are less clear, but likely relate to a lack of qualified kidney di- eticians in remote locations. Further research is required to determine the barriers to dietary care and to develop strategies to improve receipt of pre-ESRD care in all geographic locations.

While the dialysis mortality benefit from pre-ESRD care is important, kidney transplantation is the only cure for dialysis-dependent kidney disease. Our study demonstrated that better pre-ESRD care independently increases the likelihood of kidney transplantation. The factors that influence this finding are unknown, but could be related to improved participation in care, better educational resources, or avoidance of blood transfusions through use of erythropoiesis-stimulating agents. In addition, pre-ESRD care has been associated with pre-emptive kidney transplantation [24], suggesting quality CKD care could drive referrals to kidney transplant centers. PreESRD care among micropolitan and rural patients is associated with kidney transplantation similarly to urban patients, suggesting that receiving care confers a similar benefit regardless of where a patient lives.

There are important limitations to this study. First, we focused on patients with kidney disease who survived to dialysis initiation, a subpopulation of the patients with advanced CKD, which is a source for survival bias. Second, the source of data regarding pre-ESRD care was the medical evidence report (CMS 2728), which can have inconsistencies and errors, especially when completed by nonphysicians $[25,26]$. The medical evidence report, however, is the only available source of data within the USRDS to study pre-ESRD care in all adults. However, the documentation of longitudinal outcomes, including death and kidney transplantation, is more robust due to internal redundancies within the USRDS [27]. Third, we assessed geographic residence at dialysis initiation that did not account for patient movement over time, which could lead to misclassification. While every effort was made to reduce the impact of confounding, unmeasured confounders and the use of community-level surrogates for socioeconomic status may limit the interpretation of the results. Lastly, the results reported are associations and cannot imply causality.

\section{Conclusions}

There is no observed difference in the prevalence of early nephrology care or permanent dialysis access in the pre-ESRD period between micropolitan, rural, and urban dialysis patients. The protective effect of pre-ESRD care is similar across all geographic strata. Micropolitan and 
rural patients receive less dietary education than urban patients, suggesting that barriers exist to quality dietary care in remote locations.

\section{Acknowledgements}

Dr. Maripuri was supported by an institutional training grant from the National Institutes of Health (T32 DK007569). Drs. Cavanaugh and Ikizler are supported by grants from the National Institutes of Health (K23 DK080952 and K24 DK062849, respectively). Dr. Cavanaugh is also supported by the American Society of Nephrology Carl W. Gottschalk Research Scholar Grant. Funding for patient-level data, provided by the US Renal
Data System, was supported by the Vanderbilt CTSA award No. UL1TR000445 from the National Center for Advancing Translational Sciences. This paper does not represent the opinion of the US Renal Data System or the National Institutes of Health. Drs. Maripuri and Cavanaugh assume full responsibility for data management and statistical analysis. The findings of this study were presented at the Southern Society for Clinical Investigation 2012 Annual Meeting, New Orleans, La. and National Kidney Foundation 2012 Spring Clinical Meeting, Washington, D.C., USA.

\section{Disclosure Statement}

No authors have any conflicts of interest to disclose.

\section{References}

1 United States Census Bureau: Census 2000 Summary File (SF1) 100-Percent Data. Washington, United States Census Bureau, 2000.

2 Maripuri S, Arbogast P, Ikizler TA, Cavanaugh KL: Rural and micropolitan residence and mortality in patients on dialysis. Clin J Am Soc Nephol 2012;7:1121-1129.

-3 Vascular Access Work Group: Clinical practice guidelines for vascular access. Am J Kidney Dis 2006;48(suppl 1):S176-S247.

$4 \mathrm{~K} / \mathrm{DOQI}$ clinical practice guidelines for chronic kidney disease: evaluation, classification, and stratification. Am J Kidney Dis 2002; 39(2 suppl 1):S1-S266.

5 Goransson LG, Bergrem H: Consequences of late referral of patients with end-stage renal disease. J Intern Med 2001;250:154-159.

6 Chen SC, Hwang SJ, Tsai JC, Liu WC, Hwang SC, Chou MC, Lin MY, Chang JM, Chen HC: Early nephrology referral is associated with prolonged survival in hemodialysis patients even after exclusion of lead-time bias. Am J Med Sci 2010;339:123-126.

>7 Frimat L, Loos-Ayav C, Panescu V, Cordebar N, Briancon S, Kessler M: Early referral to a nephrologist is associated with better outcomes in type 2 diabetes patients with endstage renal disease. Diabetes Metab 2004;30: $67-74$.

8 Lorenzo V, Martn M, Rufino M, Hernandez D, Torres A, Ayus JC: Predialysis nephrologic care and a functioning arteriovenous fistula at entry are associated with better survival in incident hemodialysis patients: an observational cohort study. Am J Kidney Dis 2004;43: 999-1007.

-9 Arora P, Obrador GT, Ruthazer R, Kausz AT, Meyer KB, Jenuleson CS, Pereira BJ: Prevalence, predictors, and consequences of late nephrology referral at a tertiary care center. J Am Soc Nephrol 1999;10:1281-1286.
0 Obrador GT, Ruthazer R, Arora P, Kausz AT, Pereira BJ: Prevalence of and factors associated with suboptimal care before initiation of dialysis in the United States. J Am Soc Nephrol 1999; 10:1793-800.

11 Joynt KE, Harris Y, Orav EJ, Jha AK: Quality of care and patient outcomes in critical access rural hospitals. JAMA 2011;306:45-52.

12 Measuring rurality: rural-urban commuting area codes. Economic Research Service, United States Department of Agriculture. http:// www.ers.usda.gov/briefing/Rurality/RuralUrbanCommutingAreas (accessed March 8, 2011).

13 Hart LG, Larson EH, Lishner DM: Rural definitions for health policy and research. Am J Public Health 2005;95:1149-1155.

14 ZIP code RUCA approximation methodology. Washington Wyoming Alaska Montana Idaho Rural Health Research Center. http:// depts.washington.edu/uwruca/ruca-data. php (accessed March 8, 2011).

15 United States Census Bureau: Census 2000 Summary File (SF3) Sample Data: Median Household Income (P053) and Education Attainment for Population 25 Years or Older (P037). Washington, United States Census Bureau, 2000.

16 Barros AJ, Hirakata VN: Alternatives for logistic regression in cross-sectional studies: an empirical comparison of models that directly estimate the prevalence ratio. BMC Med Res Methodol 2003;3:21.

17 Cook NL, Hicks LS, O’Malley AJ, Keegan T, Guadagnoli E, Landon BE: Access to specialty care and medical services in community health centers. Health Aff 2007;26:1459-1468.

18 Rucker D, Hemmelgarn BR, Lin M, Manns BJ, Klarenbach SW, Ayyalasomayajula B, James MT, Bello A, Gordon D, Jindal KK, Tonelli M: Quality of care and mortality are worse in chronic kidney disease patients living in remote areas. Kidney Int 2011;79:210-217.
19 Jennette CE, Vupputuri S, Hogan SL, Shoham DA, Falk RJ, Harward DH: Community perspectives on kidney disease and health promotion from at-risk populations in rural North Carolina, USA. Rural Remote Health 2010;10:1388.

20 O'Hare AM, Johansen KL, Rodriguez RA: Dialysis and kidney transplantation among patients living in rural areas of the United States. Kidney Int 2006;69:343-349.

21 Clinical practice guidelines for nutrition in chronic renal failure. K/DOQI, National Kidney Foundation. Am J Kidney Dis 2000;35(6 suppl 2):S1-S140.

-22 Sullivan C, Sayre SS, Leon JB, Machekano R, Love TE, Porter D, Marbury M, Sehgal AR: Effect of food additives on hyperphosphatemia among patients with end-stage renal disease: a randomized controlled trial. JAMA 2009;301:629-635.

23 Slinin Y, Guo H, Gilbertson DT, Mau LW, Ensrud K, Collins AJ, Ishani A: Prehemodialysis care by dietitians and first-year mortality after initiation of hemodialysis. Am J Kidney Dis 2011;58:583-590.

24 Khosla N, Gordon E, Nishi L, Ghossein C: Impact of a chronic kidney disease clinic on preemptive kidney transplantation and transplant wait times. Prog Transplant 2010;20: 216-220.

25 Layton JB, Hogan SL, Jennette CE, Kenderes $\mathrm{B}$, Krisher J, Jennette JC, McClellan WM: Discrepancy between Medical Evidence Form 2728 and renal biopsy for glomerular diseases. Clin J Am Soc Nephrol 2010;5:2046-2052.

26 Kim JP, Desai M, Chertow GM, Winkelmayer WC: Validation of reported predialysis nephrology care of older patients initiating dialysis. J Am Soc Nephrol 2012;23:1078-1085.

27 Eggers PW: CMS 2728: what good is it? Clin J Am Soc Nephrol 2010;5:1908-1909. 